Original

\title{
Actividad laboral en una cohorte de pacientes con carcinoma de pulmón
}

\section{Employment in a cohort of lung cancer patients}

\section{Raquel Molina Villaverde', Jaime Feliu Batlle², Ana María Jiménez Gordo ${ }^{3}$, Belén San José Valiente}

1. Departamento de Oncología Médica. Universidad de Alcalá de Henares. Hospital Universitario "Príncipe de Asturias". Alcalá de Henares (Madrid). España.

2. Departamento de Oncología. Hospital Universitario "La Paz". Madrid. España.

3. Departamento de Oncología. Hospital de Getafe. Getafe (Madrid). España.

4. Departamento de Estadística. Hospital Universitario "La Paz". Madrid. España

Recibido: 28-11-11

Aceptado: 03-01-12

\section{Correspondencia}

Raquel Molina Villaverde

Departamento de Oncología Médica.

Hospital Universitario Príncipe de Asturias.

Carretera Alcalá-Meco s/n.

Alcalá de Henares 28805. Madrid.

TIfono.: 918878100 ext: 2363

Fax: 915791279

E-mail: mvraq@hotmail.com

ResUmen

Introducción: La actividad laboral en el cáncer de pulmón es un aspecto psicosocial que ha recibido poca atención hasta el momento actual por distintos motivos, a pesar de considerarse una dimensión de la calidad de vida para todo paciente oncológico.

Objetivos: Analizar la reinserción y adaptación al entorno laboral en una cohorte de pacientes con un carcinoma de pulmón para describir los factores que influyen en la vuelta al trabajo de estos enfermos.

Pacientes y métodos: El estudio incluyó 35 pacientes consecutivos diagnosticados de un cáncer de pulmón y que estaban empleados en el momento del diagnóstico. El cuestionario incluyó aspectos epidemiológicos, clínicos y laborales (32 variables en total) que se relacionaron con la reincorporación al mundo laboral. También se incluyeron percepciones subjetivas de los enfermos respecto a este tema.

Resultados: El 96,9\% de los pacientes pasaron a inactivos tras comenzar el tratamiento de la enfermedad y un $85,7 \%$ lo seguían estando tras éste. La presencia de secuelas fue la variable con mayor influencia en la inactividad laboral.

Conclusiones: Éste es el primer estudio exploratorio en nuestro país acerca de la reinserción laboral de los pacientes diagnosticados de un carcinoma de pulmón.

Med Segur Trab (Internet) 2012; 58 (226) 6-12

Palabras clave: Carcinoma de pulmón; Readaptación laboral; Actividad laboral.

Abstract

Background: Cancer affects many dimensions determining quality of life, including work. However, the importance of work to cancer survivors has received little attention. 
Aim: Employment and work-related disability were investigated in a cohort of lung cancer patients to describe a possible discrimination and other work issues.

Patients and Methods: The study included consecutively 35 lung cancer patients who were employed at diagnosis. The questionnaire included cancer-related symptoms and work-related factors. Clinical details were obtained from the medical record. Patients were interviewed face to face and 32 variables were recorded.

Results: 96,9 per cent of patients were unable to work after diagnosis, but $85,7 \%$ returned to work at the end of treatment. Most of the problems reported in the study were linked to the sequelae of their disease and related treatments.

Conclusions: This is the first exploratory study in Spain about labour reintegration in lung cancer patients. Further studies are necessary.

Med Segur Trab (Internet) 2012; 58 (226) 6-12

Key words: Lung cancer. Labour reinsert. Returning to work. 


\section{INTRODUCCIÓN}

Los supervivientes de cualquier enfermedad crónica han de aprender a convivir con numerosos problemas, adaptarse al estilo de vida resultante y así, organizar su vida como un proyecto de futuro a más o menos corto plazo. Con los avances en las últimas décadas, los pacientes con un cáncer cada vez se curan más y, los que no, cada vez viven en unas condiciones mejores ${ }^{1,2}$. A pesar de esto, tanto el tumor como el tratamiento específico producen secuelas y efectos secundarios que condicionan el funcionamiento físico y psíquico de la persona y le obligan a una adaptación y un cambio de comportamiento ${ }^{3}$.

Por tanto, es frecuente que se modifiquen las prioridades en la vida e incluso, se intente dar un nuevo sentido a la existencia, alterando las relaciones sociales, familiares y laborales.

Todavía el trabajo es un aspecto que ha recibido poca atención en las enfermedades neoplásicas en general, sobre todo antes de los años 70, quizás debido a la tendencia de ver el diagnóstico de un tumor maligno como el final de la vida productiva. La imagen del cáncer como una enfermedad terminal contribuyó a la falta de preocupación de los logros de orientación en el trabajo. Más recientemente, el estudio de la actividad laboral como un camino para obtener una medida de calidad de vida y un índice de si una persona es capaz o no de mantener los roles sociales en el contexto de la enfermedad, ha llamado la atención de científicos sociales y del comportamiento sobre los aspectos psicológicos de esta patología ${ }^{4}$. Y aunque la vuelta al trabajo puede ser un objetivo muy importante para los pacientes con cáncer, la Sociedad Americana de Oncología ha estimado que el $\mathbf{9 0} \%$ de los pacientes que vuelven al trabajo sienten alguna forma de discriminación 5 .

El cáncer de pulmón es uno de los tipos con mayor morbimortalidad y con un diagnóstico, en general, en etapas avanzadas de la enfermedad. Estas características conducen a que haya pocos estudios de los cambios psicosociales que sufren los supervivientes de este tumor y aún menos, en la readaptación laboral.

El principal objetivo de este trabajo es la valoración de los factores que determinan la inactividad tras el diagnóstico y la reincorporación posterior al mundo laboral de un grupo de pacientes con un carcinoma de pulmón. De forma secundaria se intentó evaluar los posibles problemas surgidos tras la reincorporación a su trabajo: relación con los superiores y compañeros y minusvalías junto a una valoración subjetiva por parte de los pacientes de su enfermedad y de la repercusión en el medio laboral.

\section{PACIENTES Y MÉTODOS}

El estudio se realizó mediante encuestas directas realizadas por pacientes diagnosticados de un carcinoma de pulmón y atendidos de forma consecutiva en la consulta de Oncología Médica. Previamente se entregó a las pacientes un consentimiento en el que se exponían los objetivos del estudio y su finalidad y el consentimiento para la utilización de los datos de la historia clínica de donde se obtuvieron los datos relativos a las características médicas de la enfermedad.

La variables estudiadas se centraban en tres aspectos fundamentales: datos demográficos (edad, estado civil, nivel de estudios), laborales (tipo de trabajo, régimen laboral, situación tras el inicio del tratamiento específico de la enfermedad, motivo y duración de dicha situación, situación laboral actual, conocimiento por parte de sus compañeros/ jefes de la enfermedad, percepciones de perjuicio laboral a causa del diagnóstico de cáncer y de dificultades en el caso de buscar un nuevo empleo) y clínicas (tipo de neoplasia, estadio, fecha del diagnóstico, comorbilidad, tipo de tratamientos empleados, secuelas de la propia neoplasia o del tratamiento empleado, intervalo libre de enfermedad, situación actual de la enfermedad). 


\section{Análisis estadístico}

La descripción de los datos cualitativos se realizó en forma de frecuencias absolutas y porcentajes y los datos cuantitativos mediante media, mediana y desviación típica (mínimo, máximo). La estimación del porcentaje de pacientes que se reincorporaron a la actividad laboral se obtuvo mediante intervalos de confianza del 95\%. La comparación de datos cualitativos entre grupos se analizó a través del test de la Chi- Cuadrado o el test exacto de Fisher según la distribución de los mismos. Los datos cuantitativos se compararon mediante el test de U Mann-Whitney. Todas las pruebas estadísticas se consideraron bilaterales y como valores significativos, aquellos $\mathrm{p}<0,05$, salvo que se especifiquen otros valores. Los datos fueron analizados con el programa estadístico SPSS 9.0.

\section{RESULTADOS}

Se recogieron treinta y cinco pacientes consecutivos, diagnosticados de un carcinoma de pulmón y que estaban empleados laboralmente en el momento del diagnóstico. La mediana de edad fue de 52,9 años (34-65). En un 94\% de los casos se trataba de hombres.

El 27\% tenían una ocupación que no requería, de forma predominante, un esfuerzo físico para su realización (las llamadas de "cuello blanco" en los estudios de Feldman ${ }^{6}$ ). El $81 \%$ eran asalariados.

En cuanto al estadio de la enfermedad en el momento del diagnóstico, un 5,7\% tenían un estadio I, un $11,4 \%$ II, un $54,3 \%$ III y un $28,6 \%$ eran metastásicos. Hasta en un $40 \%$ había otras enfermedades concomitantes, de las que predominaban la patología cardiovascular, problemas respiratorios (el más frecuente la enfermedad pulmonar obstructiva crónica) y la diabetes mellitus.

El 96,9\% de estos enfermos estuvieron de baja tras comenzar el tratamiento específico para la neoplasia y un $85,7 \%$ lo estaban en el momento actual.

El $28,6 \%$ habían sido tratados con cirugía. El $64,7 \%$ habían recibido quimioterapia y radioterapia. Un total de $88,6 \%$ habían recibido tratamiento con algún tipo de quimioterapia.

El 68,6\% tenía alguna secuela derivada de la propia enfermedad o de la modalidad terapéutica recibida, de las cuales la más frecuente fue la astenia (66,9\%).

En un $88,9 \%$ de los casos, tanto sus compañeros como sus jefes conocían el diagnóstico de su enfermedad y en el $75 \%$ no habían notado cambios por parte de éstos. El $78,9 \%$ de los pacientes pensaban que tendrían dificultad en encontrar un nuevo empleo a causa de la enfermedad pero el $76,5 \%$ no ocultaría que había tenido un cáncer en las entrevistas de otros trabajos.

\section{Correlación entre las distintas variables y la actividad laboral}

En este estudio no se observaron diferencias ni en la actividad tras el diagnóstico de la enfermedad ni en el momento de la encuesta, con respecto a las variables demográficas analizadas (género, edad, nivel de estudios). Tampoco se objetivaron diferencias en cuanto a las características laborales como el tipo de trabajo, agrupándolo en predominantemente físico o no.

En cuanto a los aspectos clínicos, la baja potencia del estudio por el número muestral no permitió detectar diferencias significativas entre la actividad laboral y el estadio de la enfermedad ( $\mathrm{p}=0.610)$ ni en relación con las enfermedades concomitantes $(\mathrm{p}=0.503)$.

Ninguno de los pacientes que tenían alguna secuela derivada de la enfermedad o del tratamiento específico estaban activos frente al $62,7 \%$ de los que no tenían ninguna $(\mathrm{p}<0.001)$.

Similar porcentaje de encuestados entre los que estaban de baja y los que no, aseguraron que sus compañeros y/o jefes sabían que tenían una neoplasia. 


\section{DISCUSIÓN}

Aunque existe un número creciente de estudios acerca de las consecuencias psicosociales del cáncer, pocos de ellos se centran en el impacto que esta enfermedad tiene en los aspectos laborales de los supervivientes. Se ha encontrado una gran cantidad de variaciones en relación al porcentaje de problemas relacionados con la situación laboral $(2,5-84 \%)^{7,8}$ y distintos factores que pueden afectar al estado funcional del superviviente y a su rehabilitación laboral ${ }^{\text {9-11. }}$.

El cáncer de pulmón constituye la principal causa de muerte por cáncer en el mundo, superando las cifras de mortalidad conjuntas del cáncer de colon, mama y próstata. El $70 \%$ de los tumores malignos pulmonares se presentan en estadios avanzados al diagnóstico donde la cirugía, única opción curativa, no es posible ${ }^{12}$. En esta situación, la quimioterapia y las terapias dirigidas constituyen la base del tratamiento aunque con resultados poco satisfactorios si los comparamos con otro tipo de tumores.

En general, hay pocos estudios realizados en supervivientes de un cáncer de pulmón al tratarse, como ya se ha mencionado, de un tumor con un bajo índice de curabilidad y con un alto porcentaje de comorbilidad asociada en forma principalmente de patología respiratoria como enfermedad pulmonar obstructiva. Suele tratarse de estudios realizados sólo en estadios localizados y, en general, se han detectado más problemas en la rehabilitación de estos enfermos a todos los niveles (incluidos los aspectos laborales) ${ }^{13} \mathrm{y}$ con una peor puntuación en los test de calidad de vida en general ${ }^{14,15}$.

Dentro de los estudios realizados específicamente sobre la readaptación laboral, encuadrando en cáncer de pulmón dentro de otros grupos de tumores, destaca uno reciente realizado en Reino Unido en el que se revisaron distintos factores que influyen en la vuelta al trabajo y objetivaron que los supervivientes de una neoplasia maligna pulmonar, del sistema nervioso central o de un mieloma múltiple tenían más problemas para su reincorporación laboral tras el cáncer ${ }^{16}$.

En otro estudio realizado en Corea basado en los datos administrativos de las bajas laborales del Registro Nacional de tumores, también se demostró que algunos tipos de cáncer tienen un efecto estadísticamente significativo en la pérdida de empleo y en el reempleo. Estos tipos incluían en carcinoma de pulmón, los tumores del sistema nervioso central y las leucemias. Este resultado estaba relacionado con mayor severidad de la enfermedad, menores tasas de supervivencia global y un mayor número e intensidad de las limitaciones físicas asociadas ${ }^{17}$.

En el año 2010, Earle y cols llevaron a cabo un estudio prospectivo de cohortes en 2422 pacientes con carcinoma de colon y de pulmón en estadios localizados y sin signos de recidiva. Los factores asociados al abandono laboral fueron significativamente más altos en el carcinoma de pulmón en comparación al cáncer colorrectal (HR 2,83). El estudio concluye que el $80 \%$ de los supervivientes con cáncer colorrectal y de pulmón son capaces de volver a trabajar; sin embargo, las personas con menos ingresos y con enfermedad en situación más avanzada son los que necesitan más apoyo ${ }^{18}$.

En el estudio de Roelen realizado en 5074 pacientes con distintos tipos de tumores, se objetivó que aquellos con un carcinoma de pulmón tenían una mayor duración de la baja laboral y sólo el $45 \%$ habían vuelto a su empleo anterior a los 2 años del diagnóstico ${ }^{19}$.

En España, hay pocos estudios sobre la reincorporación al trabajo del enfermo neoplásico en general. A falta de estudios específicos en cáncer de pulmón destacan dos realizados en diferentes tipos de neoplasias (entre las que se incluyen pacientes con carcinomas de pulmón): uno realizado en 95 enfermos ${ }^{20}$ y otro en 347 pacientes ${ }^{21}$ pero en los que, no se extraen conclusiones específicas del cáncer de pulmón por la baja representatividad de éste. En el primero de ellos se obtuvo un porcentaje de reinserción del $70 \%$ y los factores que influían de forma negativa en el proceso fueron el ser asalariado y la presencia de secuelas $^{20}$. 
En el segundo de ellos, realizado en 347 pacientes consecutivos activos laboralmente al diagnóstico y con distintas neoplasias se estudiaron la influencia de distintas variables en dos momentos claves de la enfermedad como es al inicio del tratamiento específico antineoplásico y al finalizar éste, y se recogió las percepciones subjetivas de los propios encuestados sobre distintos aspectos de su situación laboral y del entorno en el que se desarrollaba su trabajo. La mayor parte de los pacientes (85\%) estuvieron inactivos tras comenzar el tratamiento del tumor. Este hecho se relacionó en mayor medida con el sexo masculino, una edad mayor de 45 años, el tener un régimen laboral asalariado y la utilización de quimioterapia como modalidad terapéutica. Una vez terminado el tratamiento, el $41 \%$ de los entrevistados estuvo de baja laboral. En este caso, se objetivó la influencia de una edad mayor de 45 años, el tener estudios no universitarios, el realizar un trabajo predominantemente físico, la presencia de otras enfermedades concomitantes, el no conseguir una remisión completa de la enfermedad y las secuelas de la propia neoplasia o del tratamiento recibido ${ }^{21}$.

Teniendo en cuenta los resultados obtenidos, hay que insistir en el desarrollo de métodos de diagnóstico precoz y en tratamientos que, manteniendo o mejorando la eficacia, produzcan menos secuelas y en la detección de aquellas reversibles para instaurar una terapéutica apropiada y que no lleguen a ser invalidantes en un futuro más o menos próximo. Es necesaria también la atención de equipos multidisciplinarios de rehabilitación compuestos por médicos, enfermeras, fisioterapeutas, terapeutas ocupacionales, especialistas en foniatría y asistentes sociales, que tengan en cuenta los problemas individuales de cada paciente. Ya hay estudios en los que se demuestra que la atención de los profesionales sanitarios en el tema de los problemas laborales facilita la vuelta al trabajo $^{22,23}$.

\section{Limitaciones del estudio}

Se trata de un estudio exploratorio con un número bajo de pacientes para poder realizar un análisis multivariante y sacar conclusiones extrapolables a la población de enfermos con cáncer de pulmón. Por otro lado, la muestra es regional, lo que puede hacer que haya variaciones dentro de nuestro país y a nivel de otros países.

No obstante, dada la escasa cantidad de estudios desarrollados en España sobre este tema, es interesante como estudio piloto para futuros proyectos que analicen las distintas repercusiones que esta enfermedad neoplásica de tan mal pronóstico tiene en distintos aspectos psicosociales en general, y en la actividad laboral, en particular.

\section{REFERENCIAS BIBLIOGRÁFICAS}

1. Mullan F: Season of survival: reflections of a physician with cancer. New Engl J Med 1985; 313:270-3.

2. Gambosi J: Recovering from cancer: a nursing intervention programme. Oncol Nurs Forum 1990; 17(2): 215- 219.

3. García C, Fernández Y, Gómez J, Salinas P: Los supervivientes del cáncer. En: González Barón M, Ordóñez A, Feliu J, Zamora P, Espinosa E eds. Tratado de Medicina Paliativa y tratamiento de soporte en el enfermo con cáncer. Madrid: Panamericana, 1995; 1344-1358.

4. Taskila-Abrandt T, Pukkala E, Martikanen R, Karjalainen A, Hietanen P: Employment status of Finnish cancer patients in 1997. Psychooncology 2005; 14: 221-226.

5. Kurtzman SH, Gardner B, Kellner W: Rehabilitation of the cancer patient. Am J Surg 1988; 155: 791-803.

6. Feldman F: The return to work: a question of workability. In: American Cancer Society. Proceedings of the Workshop on Employment, Insurance and the Patient with cancer. New Orleans 1987; 27-35.

7. Hoffman B: Employment discrimination; another hurdle for cancer survivors. Cancer Invest 1991; 9(5): 589-595.

8. Bradley CJ, Bednarek HL: Employment patterns of long-term cancer survivors. Psychooncology 2002; 11: 188-198. 
9. Taskila T, Lindbohm ML: Factors affecting cancer survivors'employment and work ability. Acta Oncol 2007 46(4):446-451.

10. Short P, Vasey J, Tunceli K: Employment pathways in a large cohort of adult cancer survivors. Cancer 2005; 103: 1292-1301.

11. Taskila T, Martikainen R, Hietanen P, Lindbohm ML: Comparative study of work ability between cancer survivors and their referents. Eur J Cancer 2007; 43(5): 914-920.

12. Govindan R, Page N, Morgenszen D et al: Changing epidemiology of small-cell lung cancer in the United States over the last 30 years: análisis of surveillance, epidemiologic and end results database. J Clin Oncol 2006; 24: 4539-4544.

13. Schag CA, Ganz PA, Wing DS, Sim MS, Lee JJ: Quality of life in adult survivors of lung, colon and prostate cancer. Qual Life Res 1994; 3(2): 127-41.

14. Sarna L, Padilla G, Holmes C, Tashkin D, Brecht ML, Evangelista L: Quality of life of long-term survivors of non-small-cell lung cancer. J Clin Oncol 2002: 20(1): 2920-2929.

15. Schmitt R: Quality of life issues in lung cancer. New symptom management strategies. Chest 1993 ; 103 (1): 51S-55S

16. Amir Z, Brocky J: Cancer survivorship and employment: epidemiology. Occup Med 2009; 59(6): 373-7.

17. Park JH, Park EC, Park JH, Kim SG, Lee SY: Job loss and re-employment of cancer patients in korean employees: A nationwide retrospective cohort study. J Clin Oncol 2008; 26: 1302-09.

18. Earle CC, Chretien Y, Morris C, Ayanian JZ, Keating NL, Polgreen LA, Wallace R, Ganz PA, Weeks JC: Employment among survivors of lung cancer and colorectal cancer. J Clin Oncol 2010; 28: $1700-5$.

19. Roelen CA, Koopmans PC, Schellart AJ, van der Beek AJ: Resumming work alter cancer: a prospective study of occupational register data. J Occup Rehabil 2010; 14 (epub ahead of print).

20. Jiménez A, Feliu J, Molina R, Ríos E, Zamora P, González Barón M: Reinserción laboral del paciente neoplásico. Oncología 2000; 23(7): 318-326.

21. Molina R, Feliu J, Villalba A, San José B, Jiménez AM, Espinosa E, Álvarez-Mon M, González-Barón M: Employment in a cohort of cancer patients in Spain. Clinical \& Translational Oncology 2008; 10: 826-830.

22. Maunsell E, Brisson C, Dubois L, Lauzier S, Fraser A: Work problems after breast cancer: an exploratory qualitative study. Psychooncology 1999; 8: 467-473.

23. Verbeek J, Spelten E, Kammeijer M, Sprangers M: Return to work of cancer survivors: a prospective cohort study into the quality of rehabilitation by occupational physucuans". Occup Environ Med 2003; 60(5): 352-7.

|| 